\title{
ANALOG VERSUS DIGITAL DESIGN : WHEN AND WHERE TO MAKE THE CUT
}

\author{
Sayandeep Nag *
}

\begin{abstract}
System designers are frequently asked to create circuits that measure and control the analog world. One of the challenges in these types of designs is to know when to convert the signal from the analog comain to the digital domain. In the analog domain, signal conditioning techniques such as gain, offset, and filtering are used to quickly modify the incoming signal. Alternatively, mathematical algorithms are used in the digital domain to implement similar functions. Every system design is unique and requires custom solutions for each case, but there are some general guidelines that can help the designer make the right decisions concerning where to draw the line. Two circuit scenarios will be discussed in this paper. With each of the two systerns, the question of when and where to use analog signal conditioning strategies as opposed to digital techniques will be answered. The first scenario that will be used in this discussion is a simple RTD (Resistance Temperature Detectors) temperature sensing circuit. With this system, digital versus ànalog calibration, gain and offset techniques will be under evaluation. Filtering techniques is the second scenario that will be evaluated. Discussions will show that all circuit designs require a degree of analog filtering whether or not the input analog signal is DC or AC. From this premise, the advantages of analog versus digital filter designs will be investigated.
\end{abstract}

\section{Temperature and Virtues of Analog}

The most widely measured phenomenon in the process control, environment is temperature. Common elements such as RTDs, thermistors, thermocouples or diodes are used to sense absolute temperature as well as changes in temperature. Of these technologies,

*Business Development Officer, Benns Technologies, Bangalore. 
the platinum RTD temperature sensing element is the most accurate and stable over time and temperature. The RTD temperature sensing signal conditioning path that is used in this discussion is shown in Figure 1.

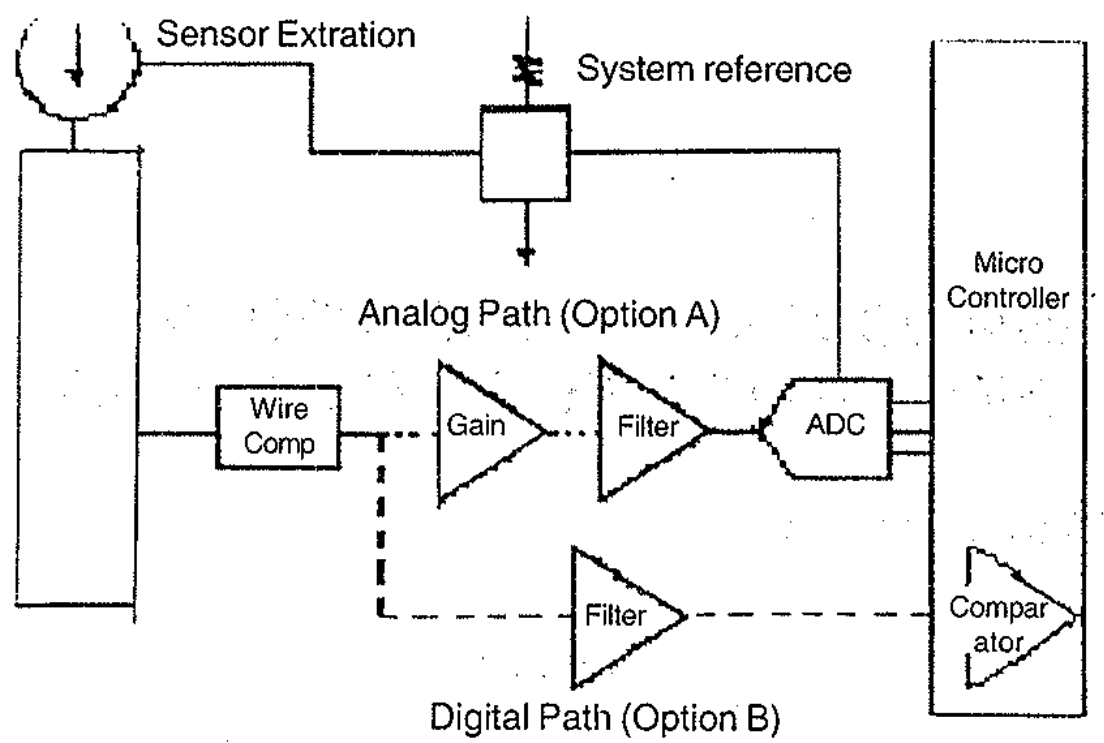

Figure 1. Block diagram of an RTD temperature sensor circuit

Typically, a data acquisition system conditions the analog signal from the RTD sensor, making the analog translation of the temperature usable in the digital domain. In this discussion, a PRTD100 will be used. The PRTD100 is a platinum RTD element that is $100 \mathrm{~W}$ at $0^{\circ} \mathrm{C}$. The temperature range that the sensor will be exposed to is $-100^{\circ} \mathrm{C}$ to $400^{\circ} \mathrm{C}$. Given this temperature range, the nominal resistance range of the PRTD 100 is $61.5 \mathrm{~W}$ at $-100^{\circ} \mathrm{C}$ to $254 \mathrm{~W}$ at $400^{\circ} \mathrm{C}$.

Since this sensor is resistive in nature, the element requires a current excitation. First impressions would indicate that any value of current source could be used to excite the PRTD element. However, if the magnitude of the current source is too high the element will dissipate enough power to self-heat. Consequently, care should be taken to insure that $\mathrm{EmA}$ of current is used to excite the RTD element. By using the system reference, ratiometric techniques can be used in this design to associate the PRTD analog current source to the A/D converter LSB size. Following the analog signal path shown in Figure 1, the voltage across the RTD element is gained and filtered before it is changed into a digital representation. If you follow the digital path, the voltage across the PRTD element is filtered and then digitized inside the microcontroller. But, prior to the implementation of either signal path, the wire resistance errors that occur as a result of temperature changes are compensated 
with a simple amplifier stage. The realization of the analog signal path portion of the block diagram of Figure 1 is shown in Figure 2 below.

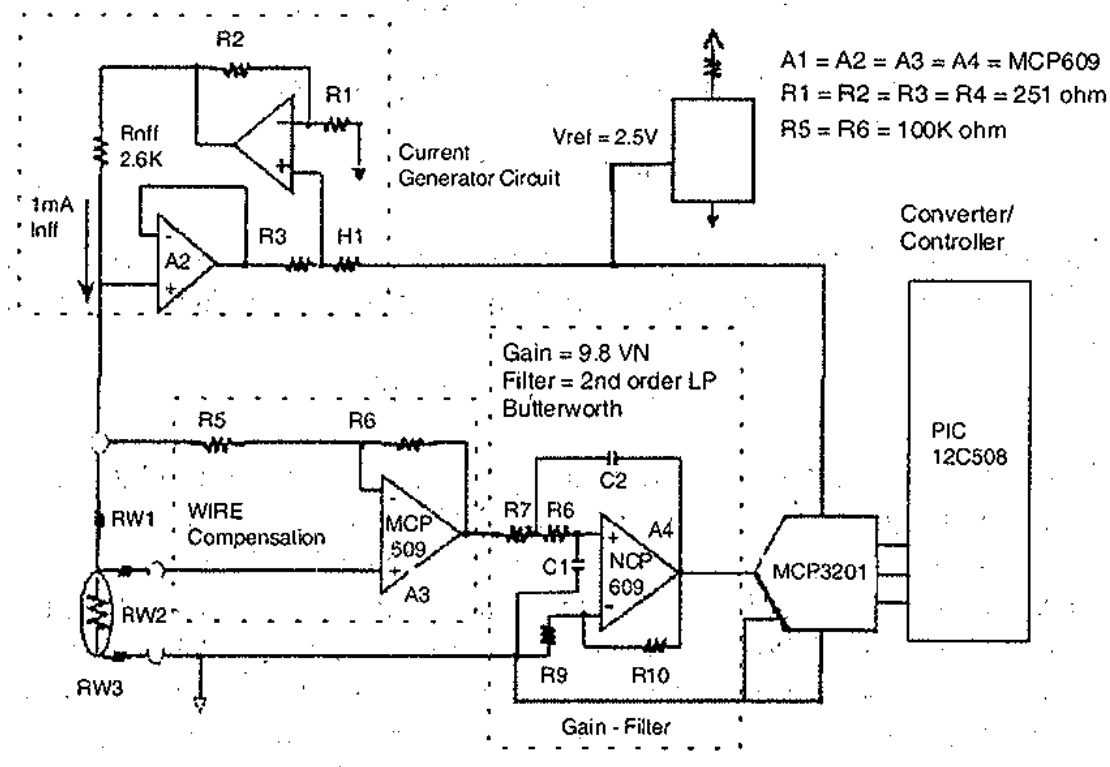

Figure 2. Full circuit realization of the block diagram of the analog singal path shown in Figure 1.

The current excitation for the RTD sensing elernent is shown in the upper left-hand corner of Figure 2. In this circuit, a voltage reference along with two operational amplifiers (A1 and $\mathrm{A} 2$ ) and several resistors are used to generate a floating $1 \mathrm{~mA}$ current source. This is accomplished by applying a $2.5 \mathrm{~V}$ precision voltage reference to $\mathrm{R} 4$. Since $\mathrm{R} 4$ is equal to $R 3$ and the non-inverting input to $A 1$ is high impedance, the voltage drop across these two resistors is equal. This amplifier (A1) along with the other three amplifier in the circuit in Figure 2 is $1 / 4$ of the equal CMOS, micropower MCP609 operational amplifier from Microchip. The voltage between $R 3$ and $R 4$ is applied to the non-inverting input of $A t$. That. voltage is gained by $(1+R 2 / R 1)$ to the output of the amplifier and the top of the reference resistor,

$P R E F$, If $R 1=R 2$ the voltage at the output of $A 1$ is equal to:

$$
\text { VOUT: } A 1=(1+R 2 / R 1) *(V R E F-V R 4)
$$

$$
\text { VOUT : } A 1=2 \text { * (VREF - VR4) }
$$

where VOUT:A1 is the voltage at the output of $A 1$ and VR 4 is the voltage drop across R4. The voltage at the output of $A 2$, VOUT: $A 2$, is equal to (VREF - VR4 - VR3). This same voltage appears at the inverting input of 
$A 2$ and across to the non-inverting input of A2. Solving these equations, the voltage drop across the reference resistor, PREF is equal to:

$$
\begin{gathered}
\text { VRREF }=\text { VOUT }: \text { A1 - VOUT }: A 2 \\
\text { VRREF }=2 \text { * }(\text { VREF }- \text { VR } 4)-(\text { VREF - VR } 4-\text { VR3 }) \\
\text { VRREF }=\text { VREF }
\end{gathered}
$$

where VRREF is the voltage across the reference resistor, RREF, and $V R 3$ is the voltage drop across R3. The current through RREF is equal to:

$$
\text { IRTD }=\text { VRREF } / \text { RREF }
$$

This circuit generates a current source that is ratiometric to the same voltage reference that is used for the AVD conversion. As was stated previously, the nominal resistance of the PRTD100 is $61.5 \mathrm{~W}$ at. $-100^{\circ} \mathrm{C}$ and $254 \mathrm{~W}$ at $400^{\circ} \mathrm{C}$. Since this resistance range is relatively low, the nominal wire resistance and its temperature coefficient to the RTD element can skew the temperature measurement. These errors can be calibrated out of the system in the digital domain given that each unit manufactured runs through is individual calibration cycle. This type of calibration cycle would require that the circuit be exposed to a known temperature bath. An alternative to implementing this calibration step in, manufacturing is a hardware analog calibration that automatically eliminates the error accurately. In order to implement the mentioned analog calibration, a three-wire RTD device is used, as shown in Figure 2, in Figure 2, the errors contributed by the wire resistances, RW1 and RW3, is subtracted from the circuit with the A3 the OP circuit. In this configuration, $R 1$ and $R 2$ are equal and relatively high. The value of $R 3(100 \mathrm{~kW})$ is selected to ensure that the leakage current is small enough through the resistor so as not to introduce errors. The transfer function of this portion of the circuit is:

$$
\text { VOUT : } A 3=(V I N-V R W 1)(1+R 6 / R 5)-V I N(R 6 / R 5)
$$

where $V I N=V R W 1+V R T D+V R W 3, V W 1$ and $V W 3$ are the voltage drops across the wires to and from the RTD and VOUT:A 3 is the voltage at the output of $A 3$. If it is assumed that $R 5=R 6=100 \mathrm{~kW}$ and $R R W 1=$ RRW3 the transfer function above reduces to:

$$
\text { VOUT }: A 3=\text { VRTD }
$$

\section{Analog Signal Path (Option A)}

Once the signal in Figure 2 is past the wire resistance compensation circuit, the choice between continuing with analog circuitry to the A/D converter or starting to migrate to the digital domain is available. If you 
remain in the analog domain, the signal path will have a low pass filter, a gain stage, followed by an A/D converter. In this analog signal path (per Figure 2) the voltage signal at the output of $A 3$ is filtered and gained with a $2^{\text {nd }}$ order, low pass filter. A low pass filter is used at this point in the circuit to reduce noise in the signal path as well as reject high frequency signals before AVD conversion. A detailed discussion of the impact of the filter function in the analog signal path is discussed in the second half of this class. The gain portion of this analog stage is used to present a voltage to the input of the AVD converter. With this gain stage, it is possible to take full advantage of the dynamic input range of the converter: This combination of function is implemented with $A 4, R 7, \mathrm{C} 1$, $\mathrm{R} 8, \mathrm{C} 2, \mathrm{R} 9$ and $\mathrm{R} 10$.

\section{Digital Signal Path (Option B)}

If you chose to migrate the signal into the digital domain earlier on, the circuit shown in Figure 3 could be used. This circuit is similar to a first order delta-sigma converter. If this circuit were compared to the classical delta-sigma converter, the integrator would be implemented with CINT. The combination of CINT, R1 and R2 also provide an analog first order low pass filter in the signal path. The comparator used is part of the controller, as well as the voltage reference. A one-bit DAC is implemented in firmware by driving RA3 in accordance with the output of the comparator, C1OUT. The firmware drives the DAC output at RA3. A digital filter is implemented with two counters and a simple averaging algorithm in the firmware.

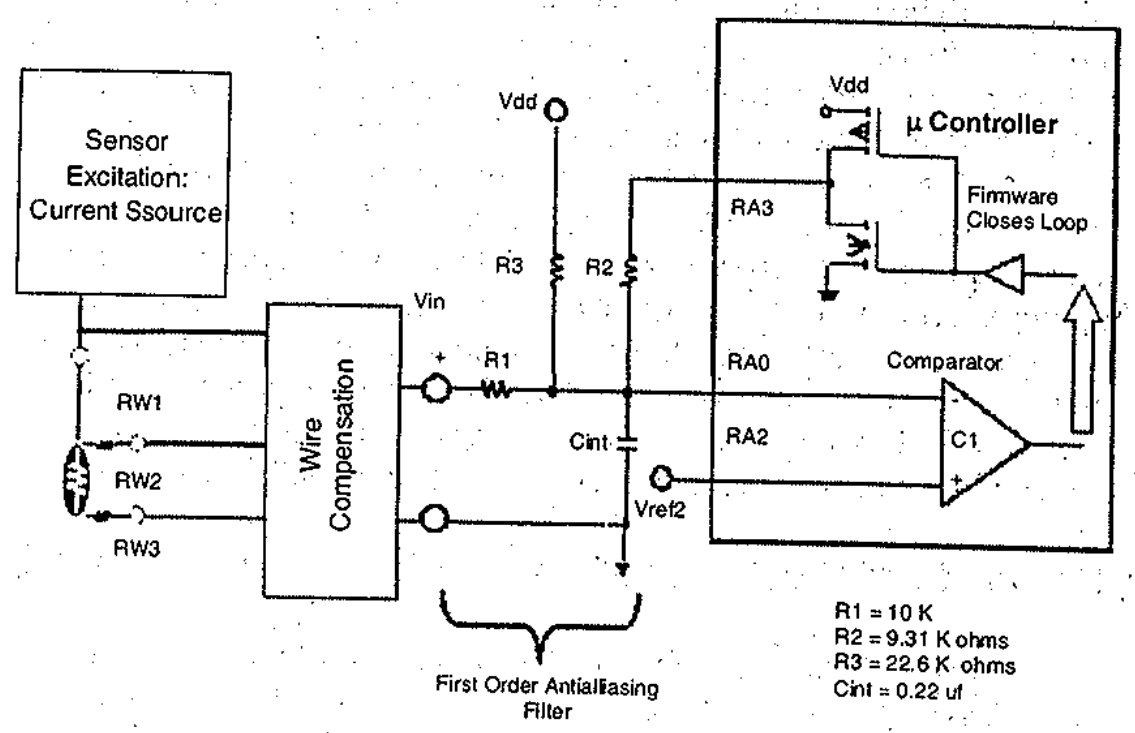

Figure 3. A first order modulator and simple digital filter is designed to implement a simple delta-sigma AND converter. 
For the circuit shown in Figure 3, any device from Microchip's PIC16C6XX MCU family can be used. The modulator section of this circuit comprises of ${ }_{R} 1 ;{ }_{R} 2,{ }_{C I N} T$, the comparator and the output terminal, RA3. When RA3 of the PIC $16 \mathrm{C} 6 \mathrm{XX}$ is set high, the voltage at RAO increases in magnitude. This occurs until the output of the internal comparator $(\mathrm{CMCON}<6>)$ is triggered low. At this point the internal driver to the RA3 output is switched from high to low. Once this has occurred, the voltage at the input to the comparator (RAO) decreases. This occurs until the comparator is tripped high. At this point, RA3 is set high and the cycle repeats. While the modulator section of this circuit is cycling, two counters are used to keep track of the time and the number of ones versus zeros that occur at the output of the comparator. This continues as long as necessary to get the required resolution.

\section{Where do you Draw the Line with this RTD System?}

With both systems, analog design is used until the wire compensation is performed. Beyond that point the trade-offs between the two systems are:

(10) Analog System: Higher speed and higher accuracy

(10 Digital System : Less components

Although the analog approach (Figure 2) has more devices in the signal path, the dynamic range of the systern can be optimized by adjusting the gain. This gain adjust can be implemented by increasing the RTD current source. Additionally, the amplifier gain stage has a direct effect of the dynamic range of the signal in respect to the input voltage range of the $A D C$ as well as the voltage reference to the ADC. Another way to improve the accuracy of this system is to carefully design the active filter. Improvements can be achieved by lowering the corner frequency or increasing the order of the filter. In contrast, the digital approach (Figure 3) has fewer discrete parts; which improves the reliability and noise resilience. However, the dynamic range is most effectively optimized by increasing the number of samples taken for each conversion result. More samples mean more time is required for the conversion.

\section{Can all Noise Problems be Solved with the Microcontroller?}

It is tempting to answer the question posed with this section with a "yes". But in all cases, it is recommended that an analog low pass filter is included in the signal path. A system that would include an analog filter or a digital filter or both is shown in Figure 4. When an analog filter is implemented it is done prior to the analog-to-digital conversion. in contrast, when a digital filter is implemented, it is done after the analog- 
to-digital conversion has occurred. It is obvious why the two filters are implemented at these particular points, however, the ramifications of these restrictions are not quite so obvious. There are a number of system differences when the filtering function is provided in the digital domain rather than the analog domain, and the user should be aware of these.

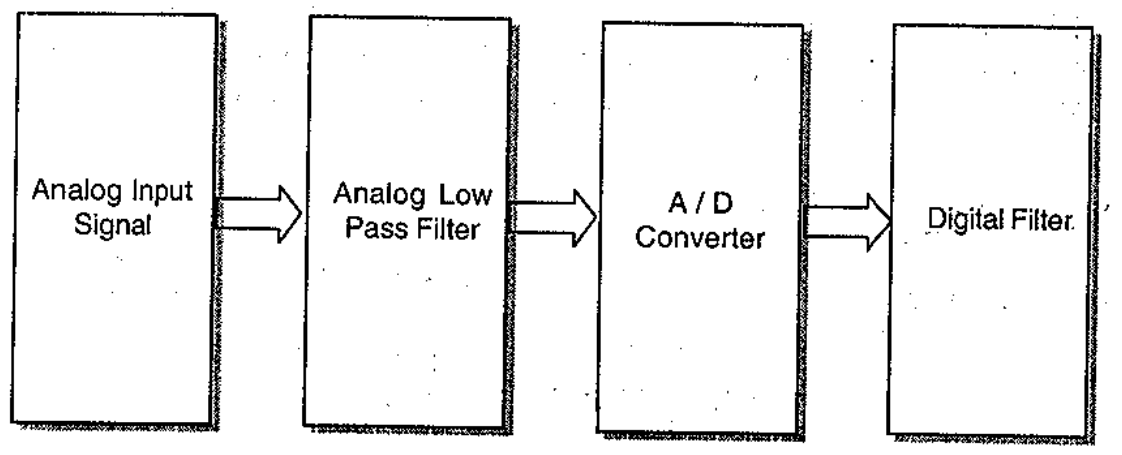

Figure 4. In system implementation, the analog filter is used before the analog-to-digital conversion and the digital fiter is inserted after this conversion has occured.

Analog filtering can remove superimposed noise before it reaches the $A / D$ converter. Most importantly, the analog filter reduces fold-back or aliasing errors from the converted results. These errors originate in the out-of band frequency region. Digital filters cannot eliminate these outof-band noise components because by the time the signal is converted the difference between the desired signal and the aliased signals is indistinguishable. The analog filter will also reduce the effects of extraneous noise peaks. Digital filtering once again cannot eliminate peaks riding on the analog signal. Consequently, noise peaks riding on signals near full scale have the potential to saturate the analog input of the AD converter. This is true even when the average value of the signal, is within limits of the system. Finally, analog filtering is more suitable for higher speed systerns, i.e. above approximately $10 \mathrm{kHz}$. In contrast, a digital filter, by definition uses oversampling and averaging techniques to reduce in-band noise. By definition, oversampling is the process of acquiring multiple conversions for a relatively static input signal. These multiple samples are then averaged, providing a more accurate representation of the signal. It is easy to see that the combination of these two processes require time to implement. Since digital filtering occurs after the $A / D$ conversion process, it can remove noise injected during the conversion process, such as quantization noise. Analog filtering cannot do this. Also, the digital filter can be re-programmed far more readily than an analog filter. Depending on the digital filter design, this gives the user the capability of programming the cutoff frequency and output data rates. 


\section{Filter Terminology}

The characteristics of filters can be described in the frequency domain as well as the time domain. The frequency and time response of a low pass filter is show in Figure 5. A low pass filter, as well as a band pass, high pass, or notch filter can be implemented in the analog as well as the digital domain. Typically, when a filter is designed in the analog domain, the frequency characteristics are first considered.

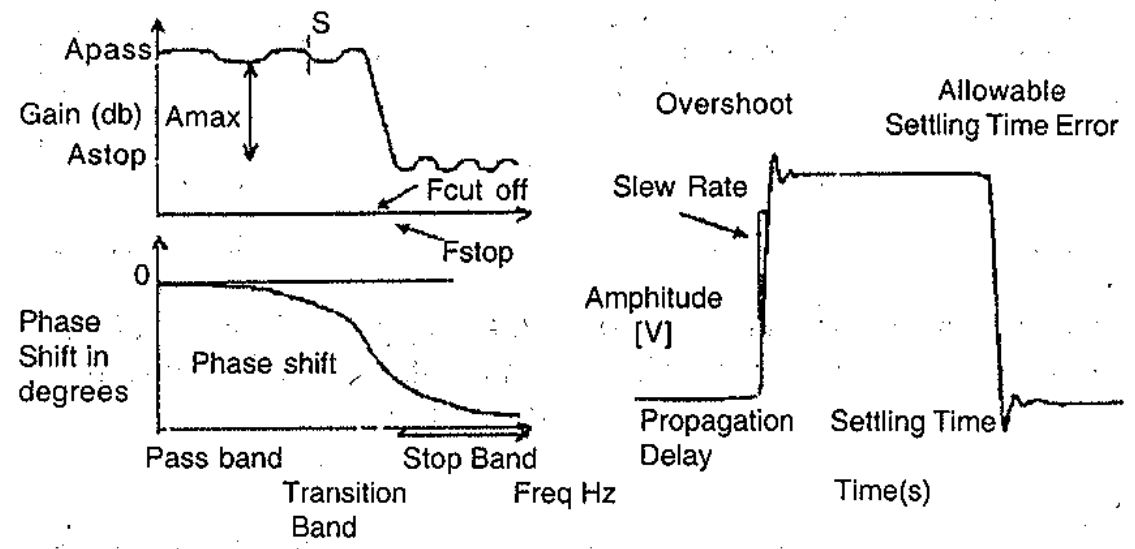

Figure 5. On the left is the frequency domain representation of a low. pass filter and on the right is the time domain representation of the same filter

For the frequency domain representation of the low pass filter shown in Figure 5, the lower frequencies are allowed through the filter up to the cutoff frequency. After the cutoff frequency, ccut.of $F$, the gain response attenuates the higher frequencies that are above the cutoff frequency. An important parameter is the filter system gain, ${ }_{\text {AMA }} X$. This is defined as the difference between the gain in the pass band region and the gain that is achieved in the stop band region or ${ }_{\text {AMAX }}=$ APASS . ASTO $P$.

In the case where a filter has ripple in the pass band, the gain of the pass band $\left({ }_{A P A S} S\right)$ is defined as the bottom of the ripple. The stop band frequency, ${ }_{\text {isto }} P$, is the frequency at which a minimum attenuation is reached. Although it is possible that the stop band has a ripple, the minimum gain $\left({ }_{A S T O} P\right)$ of this ripple is defined at the highest peak. As the response of the filter goes beyond the cutoff frequency, it falls the transition band to the stop band region. The bandwidth of the transition band is determined by the filter design (Butterworth, Bessel, Chebyshev, etc.)' and the other $(M)$ of the filter. The filter order is determined by the number of poles in the transfer function. For instance, if a filter has three poles in its transfer function, it can be described as a third order filter. When designing analog filters, the time domain response of a filter is usually 
considered second. To contrast this design approach, the time domain response of a digital filter is usually a higher priority. The graph on the right in Figure 5, can be described with five terms: propagation delay, slew rate, overshoot, settling time and settling time error. The propagation delay of a filter is the time delay that occurs from a change of the input signal to a change at the output. The propagation delay in analog filters can be directly related back to the phase response in the frequency domain. With FIR (Finite Impulse Response) digital filters, the propagation delay is caused by the amount of time required to implement the oversampling and averaging algorithms. The slew rate of this response described the rising and falling edge of the filter when responding to a step response. The overshoot and ringing phenomena of this time response to a consequence of the phase response that is shown in the Magnitude vs. Frequency response (Figure 5, graph on the left). If you will remember, the Fourier analysis of a step response (or square wave) states that you can construct a square wave by adding odd harmonic sinusoidal signals. If you re-examine the Magnitude vs. Frequency plot you will notice that the ratio of frequency to phase is not constant from lower frequencies to isro $\mathrm{p}$. Consequently the higher frequencies from the step input arrive at the output of the filter before the lower frequencies. This distortion is sometimes called group delay and is seen as ringing at the output of the filter and can be calculated with (delta phase / delta f) $/ 360$ (in seconds).. Finally, the error band that is used to define the settling time is e. For 12-bit systems this error band is usually defined as the magnitude of $1 / 2$ LSB or $0.01 \%$. For a 16 -bit system this error band is usually defined to be $0.0007 \%$.

\section{Where to Draw the Line with Filters}

Analog Filters - When designing from the frequency domain, the more common filter designs are the Butterworth, Bessel, Chebyshev, and Inverse Chebyshev (Cauer). If you are designing a low pass filter, these circuits can be designed using the filter software; FilterLAB ${ }_{T M}$ from Microchip Technology Inc. The Butterworth filter is by far the most popular design used in circuits. The transfer function of a Butterworth filter consists of all poles and no zeros. The frequency behavior has a maximally flat magnitude response in passband. The rate of attenuation in transition band is better than Bessel; but not as good as the Chebyshev filter. There is no ringing in stop band. This filter type has some overshoot and ringing in the time domain, but less than the Chebyshev or Inverse Chebyshev filters. The transfer function of the Chebyshev filter is only similar to the Butterworth filter in that it has poles and no zeros in its transfer function. In its frequency behavior there is a ripple in the passband that is determined by the specific placement of the poles in the circuit design. The rate of attenuation in transition band is steeper than Butterworth and Bessel filters. For instance, a fifth order Butterworth response is required if it is to meet the transition band width of a third order Chebyshev. Although there is ringing in the pass band region with 
this filter, the stop band is void of ringing. The step response has a fair, degree of overshoot and ringing. The transfer function of the Bessel Filter has only poles and no zeros. Where the Butterworth design is optimized for a maximally flat pass band response and the Chebyshev can be easily adjusted to minimize the transition bandwidth, the Bessel filter produces a constant time delay with respect to frequency over a large range of frequency. Mathematically, this relationship can be expressed as:

$$
C=D 1^{*} D f
$$

where $C$ is a constant, $q$ is the phase in degrees, and $f$ is frequency in $\mathrm{Hz}$ The Bessel Filter has a flat magnitude response in passband. Following the pass band, the rate of attenuation in transition band is slower than the Butterworth or Chebyshev. And finally, there is no ringing in stop band. This filter has the best step response of all of the filters mentioned above, with very little overshoot or ringing. The Inverse Chebyshev filter also has a flat magnitude response in passband. The transition region of this filter is steeper than Butterworth, Bessel, and chebyshev filters. But the steepness in the transition region creates a higher overshoot and more ringing in the time domain than other filters.

Digital Filters - Historically, the design of all of these filters originated in analog design development. This being said, all of these filters can now be implemented in the digital domain with IIR (Infinite Impulse Response) filters by using a Direct Form II Realization. When these filters are implemented in the digital arena, they are not capable of eliminating aliasing noise, but they are more flexible than the analog version in than the digital filter can be reconfigured on the fly. Digital filter design also lends itself well to being designed in the time domain. The most popular type of digital filter on the market today is the FIR (Finite Impulse Response) filter. This type of filter is fundamentally an averaging engine, providing a response that has zero phase distortion. The time domain and frequency domain representation of this filter is in Figure 6.

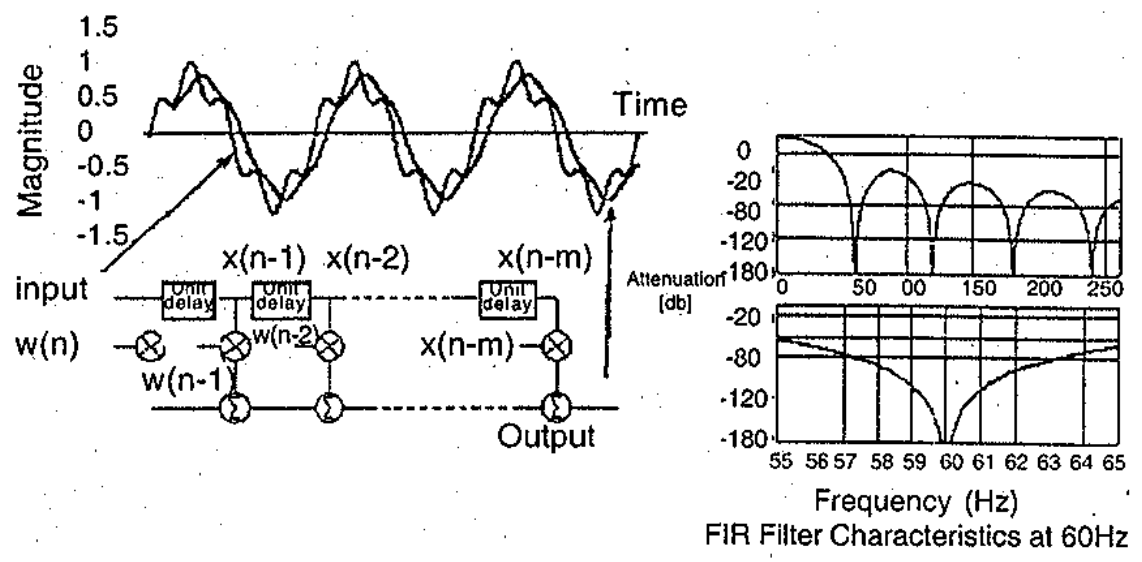

Figure 6. Time and frequency responses of a digital FIR filter. 
The phase response of the FIR filter linear so no phase distortion is created. This filer can also have a decimation filter to slow down the output data rate. The most typical digital filter that is used inside deltasigma (DS) A/D converters is the FIR filter. The DS converter combines the filtering power of the front-end modulator circuit and a $2^{\text {nd }}, 3^{\text {rd }}$ or $4^{\text {th }}$. order FIR filter to achieve bit accuracy in the low twenties. This higher accuracy is not for free. The DS converters are considerably slower that other styles of conversion, such are the SAR (Successive Approximation Register) or pipeline AVD converters.

\section{Drawing the Line}

The two application problems discussed today only scratch the surface in terms of answer to the question "When and where do you make the analog domain to digital domain cut". But from the discussion, some generalities can be brought forward: Dynamic calibration problems that are dependant on temperature or power, etc. many times can be handled most effectively in the analog domain. In this discussion, two resistors and an amplifier were used to calibrate temperature errors that were generated by the wires to the sensing element. A voltage reference was used to establish a ratiometric relationship between the sensor excitation source and the A/D converter LSB size. With both the errors it would be difficult if not impossible to calibrate out of the signal path in the digital domain. In this application, the old adage of brining the digital circuitry right to the sensor is not the most effective way of designing the circuit. Filter offers a set of considerations in the design process. When designed properly, an analog filter can remove signals and noise that is higher the $1 / 2$ nyquist. If the portion of the signal that is above before $1 / 2$ nyquist is converted it will be impossible to discriminate lower frequencies from the higher ones after the A/D conversion is complete. However, a digital filter can effectively be used to increase the signal-to-noise ratio below $1 / 2$ nyquist. This task is difficult if not impossible to perform in the analog domain. So given this limited view with two examples, the best design approach is to examine the problem from both perspectives to come up with the best solution.

\section{\#\#\#\#}

Note: The Microchip name and logo, PIC, and PICmicro are registered trademarks of Microchip Technology Inc. in the USA and other countries. FilterLab is a trademark of Microchip Technology Inc. in the USA and other countries. All other trademarks are the properly of their respective owners. 


\section{References}

1. FilterLAB, Analog Filter Program available on www.microchip.com

2. "Anti-Aliasing Analog Filters for Data Acquisition Systems", Baker, Bonnie c., AN699, Microchip Technology Inc.

3. "Making a Delta-Sigma converter with a Microcontroller", Baker, Peter, Darmawaskita, Butler, AN700, Microchip Technology Inc.

4. "Using Operational Amplifiers for Analog Gain in Embedded System Design", Baker, Bonnie C., AN682, Microchip Technology Inc.

5. "Precision Temperature Sensing with RTD Circuits", Baker, Bonnie C., AN 687, Microchip Technology Inc.

6. "Temperature Sensing Technologies", Baker, Bonnie C., AN679, Microchip Technology Inc. 